\title{
Prototype of the novel CAMEA concept-A backend for neutron spectrometers
}

Markó, Márton; Groitl, Felix; Birk, Jonas Okkels; Freeman, Paul Gregory; Lefmann, Kim; Christensen, Niels Bech; Niedermayer, Christof; Jurányi, Fanni; Lass, Jakob; Hansen, Allan

Total number of authors:

11

Published in:

Review of Scientific Instruments

Link to article, DOI:

$10.1063 / 1.5018233$

Publication date:

2018

Document Version

Publisher's PDF, also known as Version of record

Link back to DTU Orbit

Citation (APA):

Markó, M., Groitl, F., Birk, J. O., Freeman, P. G., Lefmann, K., Christensen, N. B., Niedermayer, C., Jurányi, F., Lass, J., Hansen, A., \& Rønnow, H. M. (2018). Prototype of the novel CAMEA concept-A backend for neutron spectrometers. Review of Scientific Instruments, 89(1), [015105]. https://doi.org/10.1063/1.5018233

\section{General rights}

Copyright and moral rights for the publications made accessible in the public portal are retained by the authors and/or other copyright owners and it is a condition of accessing publications that users recognise and abide by the legal requirements associated with these rights.

- Users may download and print one copy of any publication from the public portal for the purpose of private study or research.

- You may not further distribute the material or use it for any profit-making activity or commercial gain

- You may freely distribute the URL identifying the publication in the public portal 


\section{Prototype of the novel CAMEA concept-A backend for neutron spectrometers}

Márton Markó, Felix Groitl, Jonas Okkels Birk, Paul Gregory Freeman, Kim Lefmann, Niels Bech

Christensen, Christof Niedermayer, Fanni Jurányi, Jakob Lass, Allan Hansen, and Henrik M. Rønnow

Citation: Review of Scientific Instruments 89, 015105 (2018);

View online: https://doi.org/10.1063/1.5018233

View Table of Contents: http://aip.scitation.org/toc/rsi/89/1

Published by the American Institute of Physics

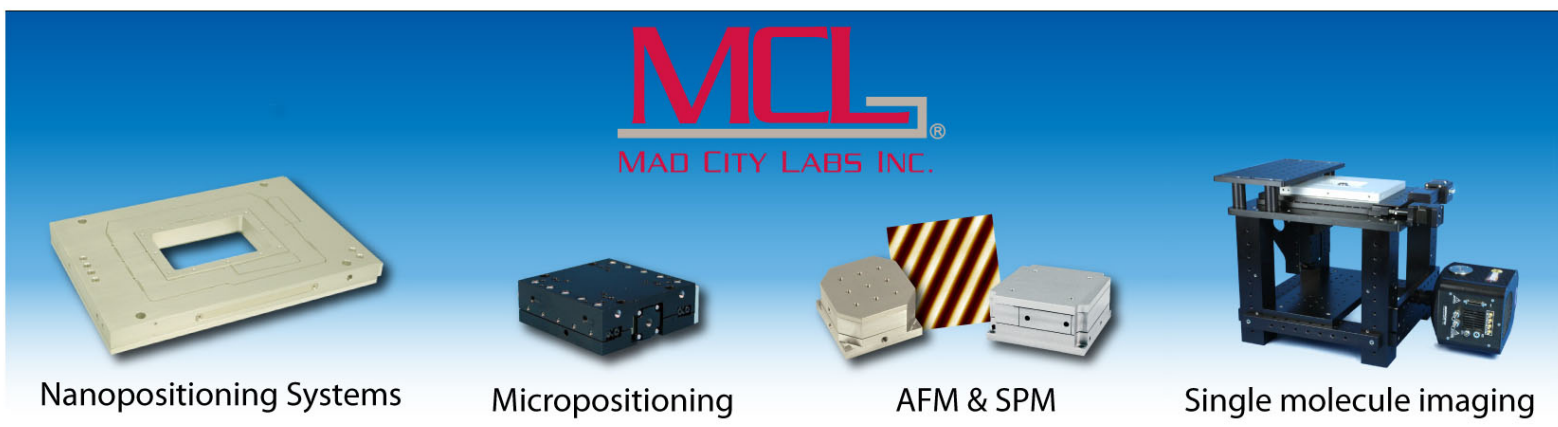




\title{
Prototype of the novel CAMEA concept-A backend for neutron spectrometers
}

\author{
Márton Markó, ${ }^{1,2, a)}$ Felix Groitl, ${ }^{1,3}$ Jonas Okkels Birk, ${ }^{4}$ Paul Gregory Freeman, ${ }^{3,5}$ \\ Kim Lefmann, ${ }^{4}$ Niels Bech Christensen, ${ }^{6}$ Christof Niedermayer, ${ }^{1}$ Fanni Jurányi, ${ }^{1}$ \\ Jakob Lass, ${ }^{4}$ Allan Hansen, ${ }^{4}$ and Henrik M. Rønnow ${ }^{3,4}$ \\ ${ }^{1}$ Laboratory of Neutron Scattering and Imaging, Paul Scherrer Institut, 5232 Villigen PSI, Switzerland \\ ${ }^{2}$ Neutron Spectroscopy Department, Wigner Research Centre for Physics, Konkoly Th. M. 29-33, 1122 Budapest, \\ Hungary \\ ${ }^{3}$ Laboratory for Quantum Magnetism, Institute of Physics, École Polytechnique Fédérale de Lausanne, \\ CH-1015 Lausanne, Switzerland \\ ${ }^{4}$ Niels Bohr Institute, University of Copenhagen, Universitetsparken 5, 2100 Copenhagen, Denmark \\ ${ }^{5}$ Jeremiah Horrocks Institute for Mathematics, Physics and Astronomy, University of Central Lancashire, \\ Preston PR1 2HE, United Kingdom \\ ${ }^{6}$ Department of Physics, Technical University of Denmark, Kongens Lyngby 2800, Denmark
}

(Received 12 April 2017; accepted 4 December 2017; published online 12 January 2018)

\begin{abstract}
The continuous angle multiple energy analysis concept is a backend for both time-of-flight and analyzer-based neutron spectrometers optimized for neutron spectroscopy with highly efficient mapping in the horizontal scattering plane. The design employs a series of several upward scattering analyzer arcs placed behind each other, which are set to different final energies allowing a wide angular coverage with multiple energies recorded simultaneously. For validation of the concept and the model calculations, a prototype was installed at the Swiss neutron source SINQ, Paul Scherrer Institut. The design of the prototype, alignment and calibration procedures, experimental results of background measurements, and proof-of-concept inelastic measurements on $\mathrm{LiHoF}_{4}$ and $\mathrm{h}-\mathrm{YMnO}_{3}$ are presented here. Published by AIP Publishing. https://doi.org/10.1063/1.5018233
\end{abstract}

\section{INTRODUCTION}

For the investigation of elementary excitations, such as phonons and magnons, inelastic neutron scattering (INS) is a well-established method. Two "standard" types of neutron spectrometers in use are direct geometry time-of-flight (ToF) spectrometers and triple-axis spectrometers (TAS). The advantage of the direct ToF spectrometer is the large coverage of momentum transfer $(\mathbf{q})$ and energy transfer $(\omega)$ space $((\mathbf{q}, \omega)$ space). This is achieved by recording neutrons with a wide range of final energies and a large coverage of solid angle with position sensitive detectors (PSDs). The drawback of the technique is the rather low incoming neutron flux since it uses a monochromatic pulsed beam. Altogether this results in an instrument that is well suited for overview studies but requires long counting times to gather sufficient statistics. This limits the number of measurements as a function of an external parameter, such as temperature, pressure, and magnetic field (parametric studies). Comparing ToF spectroscopy with the TAS technique, a typically 100 times higher incoming neutron flux is focused on the sample to investigate one specific $(\mathbf{q}, \omega)$ point. This results in much lower counting times and makes the technique well suited for parametric studies; however, long measurement times are required for mapping.

Parametric studies of low-energy collective dynamics are of particular interest for a broad variety of scientific cases. For example, in quantum magnets, high fields and pressure are used to induce quantum critical points. ${ }^{1-4}$ These studies

a)Electronic mail: marko.marton@wigner.mta.hu include the use of sample environment (SE), such as high field cryo-magnets and/or pressure cells, which limits the vertical acceptance angle of the scattered neutron beam to a few degrees. Thus, TAS are so far the instrument of choice for such measurements since they solely operate in the horizontal scattering plane. The use of SE decreases the performance of ToF spectrometers significantly by blocking a large area of the PSDs. Especially on pulsed neutron sources, the inverse ToF geometry has a big potential. This technique provides a periodic neutron beam within a defined energy range on the sample, and the scattered neutrons are sorted according to the time-of-flight, i.e., their energy. Present realizations of this technique have had some success, ${ }^{1,5,6}$ but the technique is so far only used on backscattering instruments. ${ }^{7,8}$

Since INS is in general a flux limited technique, several attempts have been made in the past to increase the detection efficiency of TAS instruments especially within the horizontal scattering plane by so-called multiplexing, ${ }^{9-20}$ where instead of a single point several points or even a constant energy line in the $(\mathbf{q}, \omega)$-space are measured. Staying within the horizontal scattering plane, this results in rather complicated mechanical solutions. Vertically scattering analyzers can cover a larger range of scattering angles; however, in all of these solutions, only one final energy is analyzed. Thus, the next logical step is to combine angular coverage with multiple final energies analyzed.

The CAMEA (Continuous Angle Multiple Energy Analysis) concept ${ }^{21,22}$ is employed in a new generation of multiplexing instruments, which utilizes the nearly perfect transmission of highly oriented pyrolytic graphite (HOPG) 


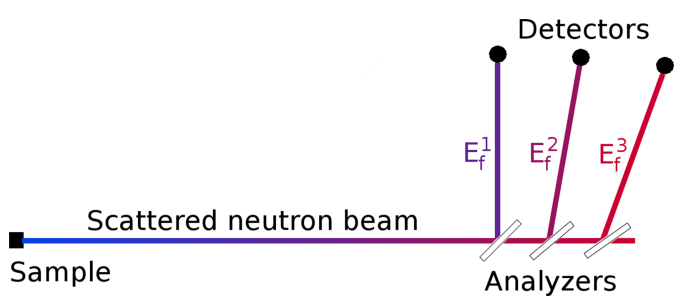

FIG. 1. A simplified sketch of the CAMEA concept. A vertical cut along one scattering angle is shown. Scattered neutrons with several different energies will be detected separately. Ideally all scattering angles should be covered continuously.

for cold neutrons ${ }^{23}$ not fulfilling the Bragg condition. This enables the implementation of a consecutive series of vertically scattering analyzer arcs all set to a different final energy (see Fig. 1). The neutrons, which are scattered out of the horizontal scattering plane, are detected by an array of PSDs with a quasicontinuous angular coverage. This results in a drastic increase of in-plane $(\mathbf{q}, \omega)$-coverage opening fast mapping possibilities and makes the design especially suited for parametric studies under extreme conditions and with small samples. Depending on the detailed design, such a CAMEA backend provides an energy and momentum transfer resolution comparable to conventional TAS and ToF instruments.

As a secondary spectrometer, CAMEA can be optimally combined with both a ToF primary spectrometer resulting in an inverse geometry ToF spectrometer and a monochromator front-end at a continuous source. The former was successfully proposed as an instrument at the European Spallation Source (ESS) where it will be installed as BIFROST. ${ }^{21}$ The latter is under construction as the new CAMEA backend to the cold TAS RITA-II at SINQ, Paul Scherrer Institut (PSI). ${ }^{22}$ Recently, other multiplexing instruments utilizing the CAMEA concept were proposed and commissioned. ${ }^{24-27}$

In the course of the BIFROST project, a prototype was built to prove the CAMEA concept. The aim of the project was to validate the concept, investigate the capabilities, and provide a solid base for the instrument design by comparing experimental results with analytical model calculations ${ }^{28}$ and Monte Carlo ray tracing simulations (McStas). ${ }^{29-31}$ To meet these challenges, the prototype was designed with flexibility to allow measurements in different settings and with easy access to each element for the installation of shielding and collimation parts. Here, the results of the prototype measurements are presented.

\section{INSTRUMENT DESCRIPTION}

The CAMEA prototype was designed by the University of Copenhagen (KU) in collaboration with the Technical University of Denmark (DTU) and PSI. The prototype was mainly constructed at DTU and installed at the spectrometer MARS (Mica Analyser Rückstreu Spektrometer) at SINQ, PSI. MARS is an inverse ToF backscattering instrument allowing for high flexibility in both energy resolution and energy range. ${ }^{32}$ The original secondary spectrometer of MARS consists of 5 analyzer-detector (inelastic) units and 6 ToF diffraction units for both positive and negative scattering angles. The units are mounted within a large tank [see Fig. 4(b)], which can be filled with argon. To provide sufficient room for the prototype, parts of the original instrument were removed. The primary and secondary spectrometers, i.e., the CAMEA prototype, are described in detail in the following paragraphs.

\section{A. Primary spectrometer}

A sketch of the MARS guide-chopper system is shown in Fig. 2. The cold source of SINQ with the guide system of MARS gives a Maxwell-Boltzmann-like spectrum at the sample position peaked at $1.5 \mathrm{meV}$ with the maximum brightness of $2.5 \times 10^{6} \mathrm{n} / \mathrm{cm}^{2} / \mathrm{s} / \mathrm{meV}$. The beam spot is $1.5 \times 5 \mathrm{~cm}^{2}$ in width and height, and the simulated horizontal and vertical divergences are $\pm 0.5^{\circ}$ and $\pm 1^{\circ}$, respectively. The primary spectrometer of MARS employs five choppers, which run at a base frequency of $f=50 \mathrm{~Hz}$. In the normal operation mode of MARS, the different tasks of the choppers are the following: the master chopper with a distance of $L=38.47 \mathrm{~m}$ to the sample position shapes the pulse. The first and the third choppers avoid an overlap of subsequent pulses, while the last two choppers close to the sample position are the order sorting choppers separating the signal from different analyzer reflections in time. In order to improve the energy resolution, the master chopper can be operated at $f=50 \times n \mathrm{~Hz}$ with a maximum of $n=7$. Details on the choppers are given in Table I.

MARS was optimized for using the (002) and (006) reflections of the mica analyzers $\left(d_{(002)}=9.99 \AA\right)$ close to backscattering geometry. Therefore, the third chopper and the order selecting choppers (\#4 and \#5) allow for maximum a third part of the time window, which restricts the wavelength band on the sample. This way at $50 \mathrm{~Hz}$ base frequency the first and second order reflections from the mica analyzer arrives at the detector at separate times.

To match the energy resolution of the CAMEA backend, the chopper system was modified. In the course of the experiments, a base frequency of $f=14 \mathrm{~Hz}$ was used. This resulted in a pulse duration of $0.605 \mathrm{~ms}$ and a repetition time of $71.4 \mathrm{~ms}$. Thus, taking into account the order sorting choppers, the initial wavelength band was $2.45 \AA$. Due to the lack of efficiency under the given conditions (low flux, small covered scattering angle, much lower duty cycle), order sorting was not used for

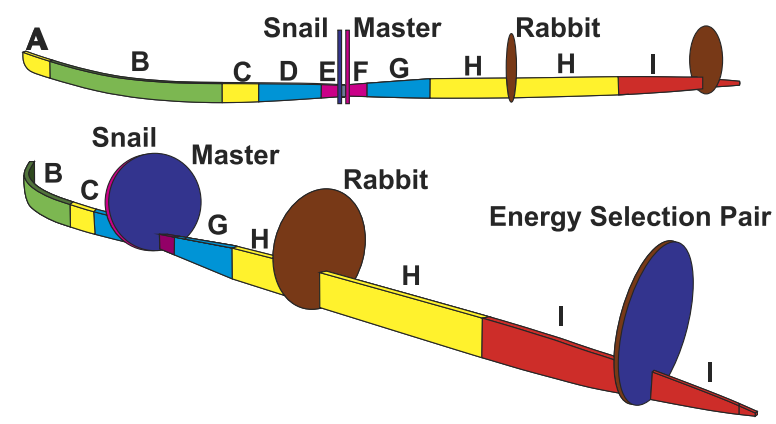

FIG. 2. Sketch of the primary spectrometer of MARS showing the different guide sections of the instrument. The curved B-section avoids direct line of sight reducing the background, while the converging (D and $\mathrm{E}$ ) and divergent ( $F$ and $G$ ) sections temporarily reduce the beam cross section at the leading chopper to increase the time (energy) resolution of the spectrometer. The converging section I focuses the beam at the sample position. Sections A, C, and $\mathrm{H}$ are standard straight mirrors. ${ }^{32}$ 
TABLE I. Choppers of MARS. Positions are given with respect to the master chopper (\#2, bold). The frequency multiplicator of the master chopper is $n=$ $1, \ldots, 7$.

\begin{tabular}{lcccc}
\hline \hline No. & Name & Position (m) & Opening angle (deg) & $f(\mathrm{~Hz})$ \\
\hline 1 & Snail & -0.308 & 4.05 & 50 \\
$\mathbf{2}$ & Master & $\mathbf{0}$ & $\mathbf{3 . 0 5 3}$ & $\mathbf{5 0 n}$ \\
3 & Rabbit & 15.601 & 54.27 & 50 \\
4 & Energy window & 34.439 & 121.02 & 50 \\
5 & Energy window & 34.494 & 121.02 & 50 \\
\hline \hline
\end{tabular}

the CAMEA prototype. However, due to the specialized design of the chopper cascade, there is no possibility to use more than one-third of the full beam.

During the operation, the need of intermediate energy resolution could be solved by a slight dephasing of the first chopper. This resulted in a shorter, wavelength dependent pulse duration with a small, wavelength dependent time shift. In the case of a required higher primary resolution, a phase shift of $3^{\circ}$ was applied. Here, the pulse is shaped by both the first and the second choppers: the beginning and the end of the pulse are defined by the opening of the master chopper and the closing of the snail chopper, respectively (see Fig. 3). Compared to the original pulse width, the phase shift reduces the effective pulse width to $45 \%$ at the beginning of the wavelength band and to $60 \%$ at the end of the wavelength band. Throughout this paper, this mode is referred to as high resolution mode, while the original mode without a dephasing of the first chopper is called low resolution mode.

The primary energy resolution when operating at $14 \mathrm{~Hz}$ is $\Delta E_{p}=2 E_{p}^{1.5} 6.42 \times 10^{-3}(\mathrm{meV})$ giving $0.07 \mathrm{meV}, 0.20 \mathrm{meV}$, and $0.57 \mathrm{meV}$ resolutions (FWHM) at $5 \mathrm{meV}, 10 \mathrm{meV}$, and $20 \mathrm{meV}$ primary energies, respectively. In high resolution modes, as it is described earlier, the resolutions are $40 \%-60 \%$ of the given numbers depending on the position in the wavelength band. The resolution and also the time-shift caused by the dephasing were always calculated numerically by calculating the position and shape of the pulse just after the master chopper. The incident neutron spectrum at the sample has a peak at around $1.5 \mathrm{meV}$ with the flux of $2.5 \times 10^{6} \mathrm{n} / \mathrm{cm}^{2} / \mathrm{s} / \mathrm{meV}$; the flux at $4 \AA$ is around $1.3 \times 10^{6} \mathrm{n} / \mathrm{cm}^{2} / \mathrm{s} / \mathrm{meV}$.

\section{B. Secondary spectrometer: The CAMEA prototype}

The actual prototype for the CAMEA concept [see Fig. 4(b)] replaced part of the secondary spectrometer of MARS. The prototype covers only a small angular range compared to a "real" instrument, e.g., $60^{\circ}$ (CAMEA), $77.5^{\circ}$ (MultiFLEXX), and $90^{\circ}$ (BIFROST). Here, the prototype is centered to a fixed sample scattering angle of $2 \Theta=-60^{\circ}$. The prototype itself has an aluminum frame structure [see Fig. 4(a)], which is divided in two parts. The bottom part is fixed to the floor, while the upper part is movable in the radial direction allowing for easy access to every part of the prototype.

The upper frame contains the 3 analyzer modules and 3 detector modules and supports the shielding. In order to allow for a flexible investigation of the CAMEA concept, the frame was designed such that the analyzer modules could be moved

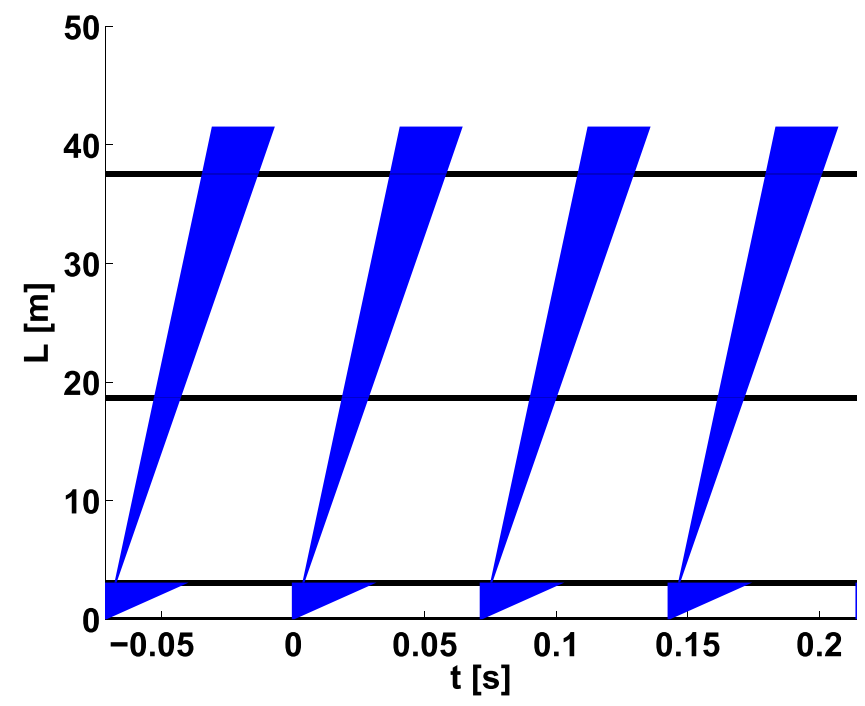

(a)

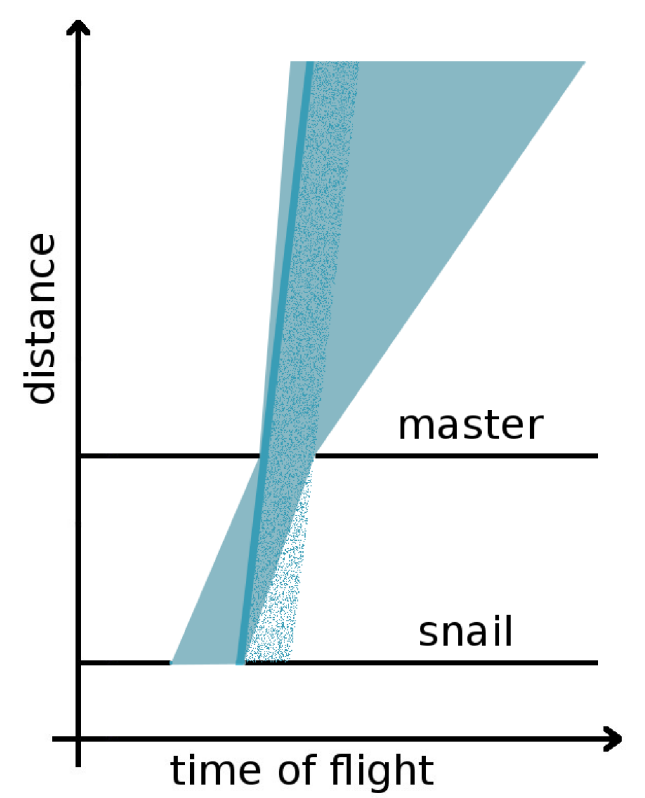

(b)

FIG. 3. ToF diagram of the primary spectrometer of MARS. (a) The full ToF diagram. The duty cycle is $1 / 3$ due to order sorting. (b) Schematic ToF diagram of the first two choppers displaying the effect of a dephasing of the first chopper. The deep blue area represents the possible trajectories of the neutrons passing through the choppers, while the light blue line represents the trajectories of neutrons having the same energy. The dotted area indicates the extended path lengths in the normal setup. Note that, for a given instrument geometry, the relative energy resolution of the primary instrument is directly proportional to the pulse duration.

radially in between 80 and $240 \mathrm{~cm}$ distance from the sample, while the detectors could be moved radially $100-240 \mathrm{~cm}$ from the vertical sample axis and in height between $-78.4 \mathrm{~cm}$ and $-180 \mathrm{~cm}$ with respect to the sample height. The movement possibility enables the realisation of the different Rowland focusing geometries including the asymmetric geometries (see Fig. 5). In addition, the aluminum profile structure of the frame allows for a flexible mounting of shielding. 


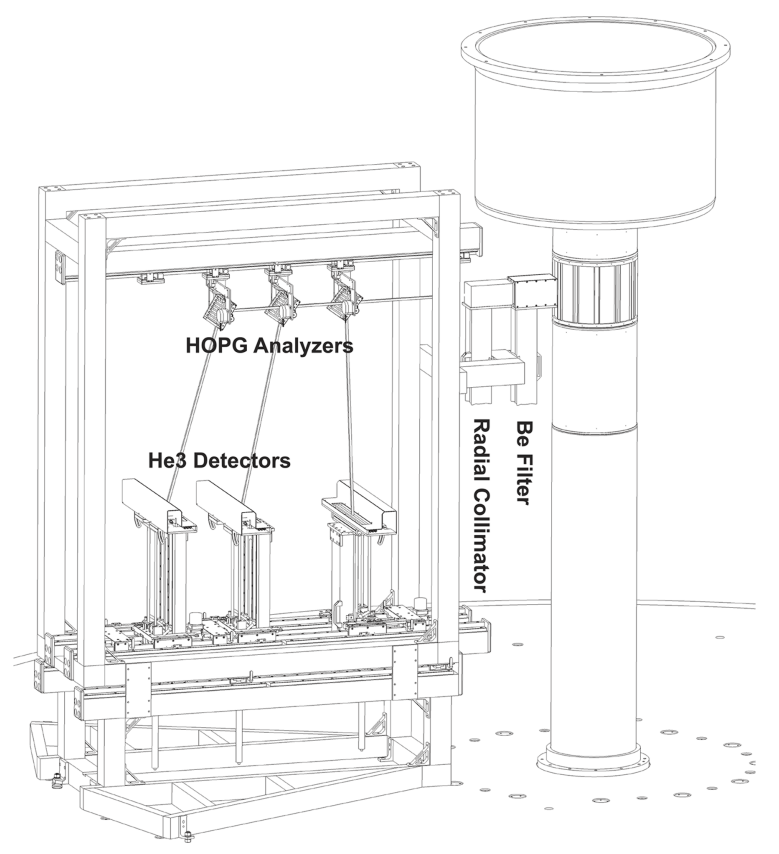

(a)

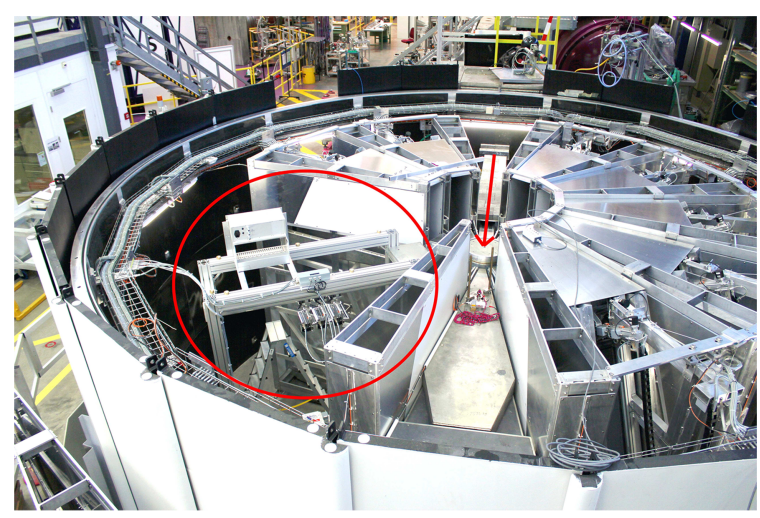

(b)

FIG. 4. (a) Sketch of the CAMEA prototype consisting of two frames. The bottom frame is fixed, while the upper frame is radially movable. The upper frame contains 3 analyzer modules and 3 detector modules and provides a structure of shielding (not shown here). The flexible design of the frame allows a radial movement of the analyzer modules and a radial and vertical movement of the detector modules. (b) The Prototype in the MARS tank. The red arrow displays the direction of the incident beam, and the red circle shows the prototype centered at a scattering angle of $2 \Theta=-60^{\circ}$.

The $15 \mathrm{~cm}$ active width of analyzer blades limits the range of the visible scattering angle of the sample between $\Delta 2 \theta=3.5^{\circ}$ and $10.7^{\circ}$. The finite dimensions of the aluminum frame and the finite maximal height of the detector limit the analyzer scattering angles resulting in a final energy range from $1.81 \mathrm{meV}$ to $32 \mathrm{meV}$. The beryllium filter and the radial collimator are optional parts of the prototype; during the measurements shown here, they were not in use. Their effect on the quality of the measured data is investigated in Ref. 26.

\section{Analyzer modules}

The CAMEA concept uses the so-called Rowland focusing geometry. ${ }^{33}$ This geometry focuses neutrons scattered

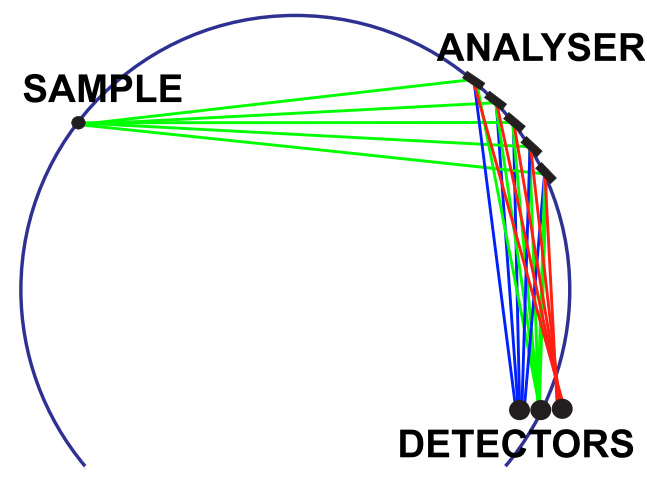

(a)

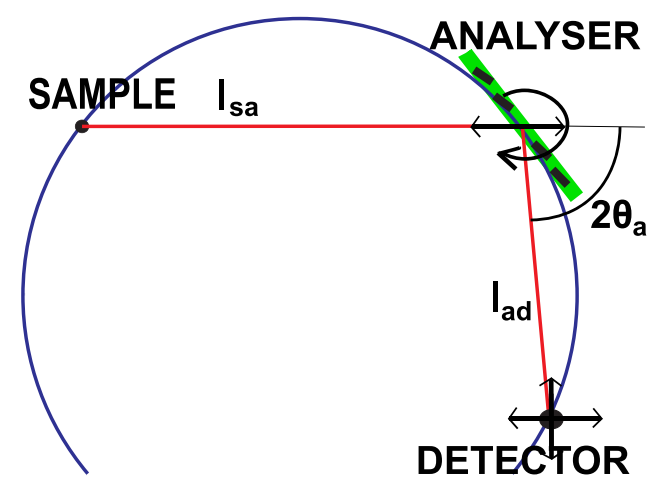

(b)

FIG. 5. Analyzer-detector assembly. (a) Prismatic analyzer. The outer detectors are not in the optimal positions, but this arrangement enables a simple mechanical solution. Blue, green, and red lines show the smaller, nominal, and larger final energies, respectively. (b) Movement possibilities of one analyzerdetector batch. The frame of analyzer crystals is shown by a green stripe. Each analyzer crystal can be rotated by hand (not marked). For simplicity, only the middle detector of the three tubes is shown.

by the same energy and scattering angle, but in different vertical angles, to one point by placing the source (in this case the sample), the analyzer crystals, and the center of the detector on a Rowland-circle [see Fig. 9(a)]. The plane of the frame holding the analyzers touches the Rowland circle at the middle analyzer blade. The radius of the Rowland circle is some meters; thus, the effect of the non-zero distance between the positions of the outer analyzer blades and the Rowland circle on the energy resolution is not measurable. To fulfill the focusing condition, each analyzer shall reflect a beam of the nominal analyzer energy, arriving from the centre of the sample to the centre of the middle detector. This is enabled by the individual rotation possibilities of the analyzer blades. Any change in distances (sampleanalyzer or analyzer-detector) and/or analyzer energy requires an adjustment of the individual rotation angles of the analyzer blades. This is easily realized with the highly flexible analyzer modules.

The analyzer modules were mounted at beam height. Each module consists of an aluminum frame, which holds up to 7 Si (100) wavers serving as support for the HOPG analyzer crystals purchased from Panasonic Corporation, Japan (see Fig. 6 and Table II). The crystals are fixed on the Si using 


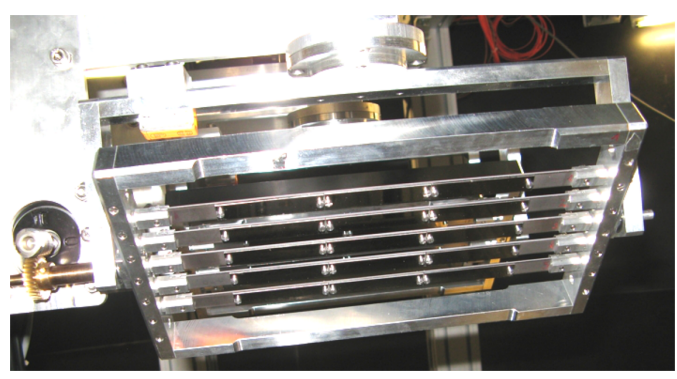

FIG. 6. Analyzer frame of the CAMEA prototype. The vertically and horizontally rotatable frame holds rotatable $\mathrm{Si}$ wavers which serve as holders for the HOPG.

aluminum screws. Since the high and low mosaicity crystals have different lengths, the silicon wafers have several holes to be able to fix three shorter or two longer crystals on them resulting in $15 \mathrm{~cm}$ active length. Only one type of crystal was used in one analyzer frame. For different frames, different crystals were used since as the previous calculations showed and the measurements described later proved, the mosaicity of the crystals have no measurable effect on the resolution. Two alternative fixation methods are possible, as has been tested recently. ${ }^{34,35}$ For alignment, the module frame can be manually rotated around the vertical axis while the rotation around the horizontal axis perpendicular to the scattered neutron beam is motorized. Within the frame, the individual wafers can be rotated manually in order to realize different focusing geometries.

\section{Detectors}

To achieve a useful q-resolution, position sensitive detectors (PSDs) are used. The orientation of the detectors is perpendicular to the scattering plane of the analyzers [see Fig. 4(a)]. Thus, each pixel along the tube can be converted into a different sample scattering angle $(2 \Theta)$. The CAMEA concept may use the prismatic analyzer concept. ${ }^{36}$ Here, the energy resolution is determined by distance collimation. In combination with a relaxed analyzer mosaicity, this allows adjacent detector tubes to collect neutrons with slightly different final energies scattered from the same analyzer.

Therefore, each detector unit of the prototype consists of three cylindrical PSDs which are mounted next to each other (see Fig. 7). The ${ }^{3} \mathrm{He}$ detector tubes were purchased from

TABLE II. Description of HOPG batches. Badges \#2 and \#3 can be combined to obtain a single large analyzer for experiments with this requirement. For price-performance optimization, crystals with different mosaicities were purchased and tested. All purchased crystals had a width of $10 \mathrm{~mm}$ and a thickness of $1 \mathrm{~mm}$.

\begin{tabular}{lcccc}
\hline \hline Batch no. & $\begin{array}{c}\text { Nominal mosaicity } \\
(\operatorname{arc} \text { min })\end{array}$ & $\begin{array}{c}\text { Measured mosaicity } \\
(\operatorname{arc} \text { min })\end{array}$ & $\begin{array}{c}\text { Number } \\
\text { of pieces }\end{array}$ & $\begin{array}{c}\text { Length } \\
(\mathrm{mm})\end{array}$ \\
\hline 1 & 24 & 32 & 15 & 50 \\
2 & 30 & 38 & 15 & 50 \\
3 & 30 & 38 & 15 & 50 \\
4 & 60 & 79 & 10 & 75 \\
5 & 90 & 118 & 10 & 75 \\
\hline \hline
\end{tabular}

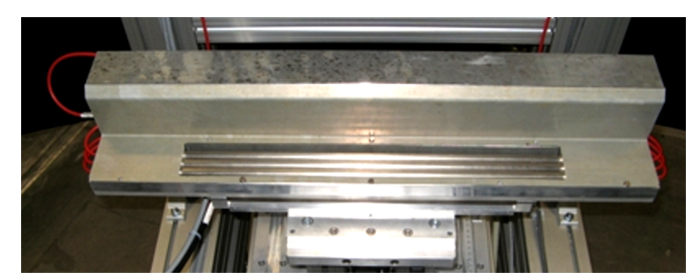

FIG. 7. Detector unit of the prototype. Three $0.5^{\prime \prime}$ position sensitive tubes are mounted parallel in the holder.

Reuter-Stokes and had a diameter of $0.5^{\prime \prime}(1.26 \mathrm{~cm})$, a length of $50 \mathrm{~cm}$, a fill pressure of 10 bars, and a position resolution of $0.5 \mathrm{~cm}$. Initially, the tubes were placed in the horizontal plane.

\section{Shielding}

The original backend shielding of MARS consists of borax water tanks surrounding the secondary instrument, borated plastic covering the inner wall of the tank, and gadolinium painted walls between each analyzer-detector unit. During the installation of the prototype, three inelastic units with their Gd shielding were removed to create enough space for adjusting the prototype [see Fig. 4(a)]. The large amount of free air around the prototype in combination with the borated polyethylene at the back of the prototype (serving as a good reflector) were the major source of background with the absence of any extra shielding on the prototype frame. Therefore, additional $\mathrm{Cd}$ shielding around the aluminum frame and between the different analyzer-detector units was installed to eliminate this part of the background. Furthermore, the analyzer frames, motors, and detector holders were covered with Cd masks.

The second largest source of background at MARS is the scattering of the incident (cold) neutron beam around the sample region. This can be blocked by temporary slits, e.g., by using $\mathrm{Cd}$. The resulting secondary gamma radiation can be well discriminated by the detector electronics. The final solution to eliminate the air scattering around the sample was an additional evacuated cylindrical sample chamber with the radius of $20 \mathrm{~cm}$. This sample chamber was covered with $\mathrm{Cd}$ above and below the scattering plane. The top of the sample chamber was designed such that it is attachable to a closed cycle refrigerator head to be able to cool the samples. Note that for inelastic measurements an orange cryostat was used without this sample chamber. The effect of the shielding and results of different measurements are discussed in Sec. IV B.

\section{Energy resolution}

The energy resolution of the prototype was investigated by carrying out several measurements with different instrument configuration.

The energy resolution of the analyzer-detector-unit for a general case is

$$
\Delta E_{f}=E_{f} 2 \cot \left(\theta_{a}\right) \Delta \theta_{a},
$$

where $E_{f}$ is the final energy and $\theta_{a}$ is the scattering angle of the analyzer. $\Delta \theta_{a}$ can be calculated by using the basic idea of 
Popovici, ${ }^{37}$

$$
\begin{aligned}
\left(\Delta \theta_{a}\right)^{2} & =C^{2} \frac{d^{2}+s^{2}+m^{2}\left(l_{s a}-l_{a d}\right)^{2}}{\left(l_{s a}+l_{a d}\right)^{2}} \\
& -C^{2} \frac{d^{2} l_{s a}-s^{2} l_{a d}+2 m^{2} * l_{s a} l_{a d}\left(l_{a d}-l_{s a}\right)}{\left(l_{s a}+l_{a d}\right)^{2}\left(a^{2}\left(l_{s a}+l_{a d}\right)^{2}+d^{2} l_{s a}^{2}+s^{2} l_{a d}^{2}+4 m^{2} l_{s a}^{2} l_{a d}^{2}\right)},
\end{aligned}
$$

where $s$ is the sample size, $a$ is the effective analyzer height (total analyzer height multiplied by $\sin \theta_{a}$ ), $d$ is the width of the detector, $m$ is the mosaicity, $l_{s a}$ and $l_{a d}$ are the sampleanalyzer and analyzer-detector distances, respectively, and $C$ is the ratio between the Gaussian-equivalent FWHM and the width of a box function, $C^{2}=(8 \ln (2)) / 12 \approx 0.46$. The detector $\left(0.5^{\prime \prime}\right.$ cylindrical tube) has likewise a close to boxlike efficiency distribution perpendicular to the tube axis due to the 10 bars ${ }^{3} \mathrm{He}$ fill pressure and the low analyzed energy.

Neglecting the effect of mosaicity, the resolution can be calculated with a simplified approach,

$$
\left(\Delta \theta_{a}\right)^{2}=C^{2}\left(\frac{s^{2}}{l_{s a}^{2}}+\frac{a^{2}\left(l_{s a}-l_{a d}\right)^{2}}{l_{s a}^{2} l_{a d}^{2}}+\frac{d^{2}}{l_{a d}^{2}}\right) .
$$

The differences between Eqs. (2) and (3) were less than 3\% in all of our measurements. In the symmetric case $\left(l_{a d}=l_{s a}\right)$, the difference is exactly zero; in other cases, Eq. (2) gives a more precise resolution than Eq. (3).

\section{ALIGNMENT AND CALIBRATION}

\section{A. Characterization of HOPG crystals}

The HOPG crystals were mounted and aligned optically within the frame, and the orientations and mosaicity of the crystals were checked with neutrons using the ToF diffractometer POLDI ${ }^{38}$ and the two-axis neutron diffractometer MORPHEUS at SINQ, PSI. The POLDI instrument is designed as a strain scanner [see Fig. 8(a)] but can be used as a 1D Laue camera to characterize the mosaic spread of HOPG crystals. The sample is illuminated through a direct line of sight to the thermal moderator of the SINQ source. Neutrons scattered from the sample are measured by a $1 \mathrm{D}$ position sensitive detector. Thus, using the white beam, the peak and the tails of the reflection are measured at once through the channels of the PSD.

Since HOPG is a strong scatterer, only a few seconds are required to measure the reflection with sufficient statistics. In total, 3 frames of the prototype were investigated. Here, only the results for the horizontal mosaic spread of one frame (mosaic spread of $30^{\prime}$ ) are shown as an example. A more detailed discussion of the results can be found in Ref. 39. The crystals of a frame were scanned with a $2 \times 10 \mathrm{~mm}^{2}$ slit setting [see Fig. 8(b)]. A Gaussian was fitted to the data, and the result of the FWHM angular widths for each of the scans is shown in Fig. 8(c). The single blades of the frame are separated by dashed red lines, while the crystals of each blade are separated by black dashed lines. The variations in mosaicity between the crystals are small. The FWHM increases toward the edges of the crystals where holes for fixation have been
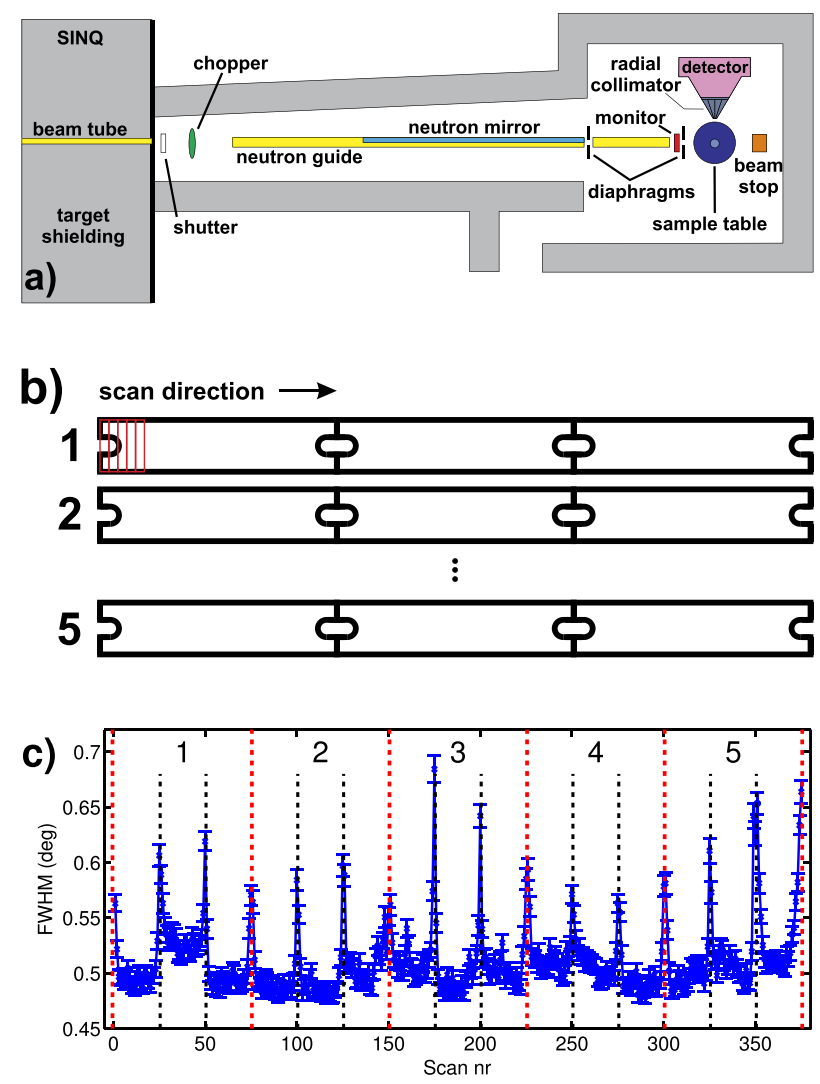

FIG. 8. (a) Layout sketch of the POLDI instrument at SINQ, PSI (b) Schematic illustration of the HOPG scan procedure in the horizontal setting. (c) Fit results of the FWHM angular spread of the single scan points in horizontal orientation for one prototype frame. The 5 blades of the frame are separated by red dashed lines, while single crystals are separated by black dashed lines. Around the edges of the crystals, the mosaic spread is deteriorated due to the milling of holes.

milled into the crystals. By doing this, the mosaic spread near the holes was deteriorated. This effect has also been observed using energy-selective neutron imaging, ${ }^{40}$ another promising method to quickly investigate a large number of crystals. The observation of the deterioration of the mosaicity around the holes led to the investigation of different mounting methods for future CAMEA-type spectrometers. ${ }^{34,35}$

\section{B. Alignment of backend}

Since for the different measurement geometries, the corresponding optimal positions and angles according to the Rowland geometry are different, the analyzer is needed to be aligned after each change in geometry. The procedure was as follows: The upper frame was pushed backwards, allowing to mount a laser at an effective sample position. Due to spatial restrictions, this effective sample position was $10 \mathrm{~cm}$ closer to the prototype frame than the actual sample position. In order to ensure the correct distances, the alignment had therefore to be done with a $10 \mathrm{~cm}$ offset, which was corrected afterwards. Starting with the outermost analyzer frame, the frame was rotated into the calculated orientation. Each analyzer blade was then rotated such that the laser beam was reflected onto the middle detector tube. In order to check the final alignment of one analyzer frame, the law of optical reciprocity was used: 


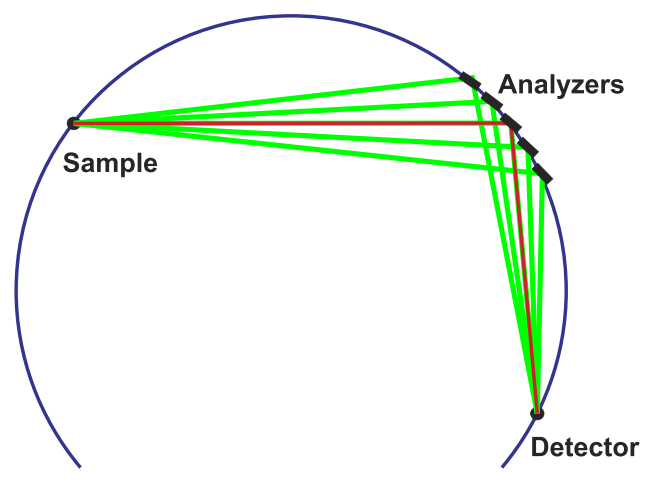

(a)

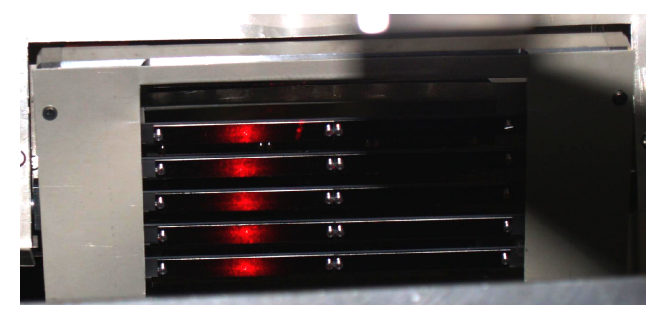

(b)

FIG. 9. Checking the alignment of the analyzer frames. (a) Way of the laser in the Rowland geometry. Blue circle is the Rowland circle; red line is the way of light from the laser source to the detector; green lines show the way of the light reflected back by the detector tube. (b) Well aligned analyzer frame. The analyzer batch focuses back the light reflected from the detector tube. The camera is next to the laser.

The laser beam starting from the sample position hits one HOPG sheet and got reflected by the cylindrical detector tube. Using the tube as a secondary light source, it was checked whether it reflects on the other crystals and comes back to the sample position [see Fig. 9(b)]. This alignment procedure was applied to all three analyzer frames. As the last step, the detector and analyzer positions were corrected for the $10 \mathrm{~cm}$ offset and the upper frame was moved back into position.

\section{Calibration}

The calibration itself consists of two parts. First, the energies of the analyzers have to be calibrated using the vanadium standard. Since both the primary and the secondary flight paths are known, the energy can be calculated from the flight time. A difference between the calculated and the measured energies was experienced, if the sample was not well centered vertically. The accuracy of the vertical alignment with precise knowledge of distances depends only on the measurement time. A standard vanadium sample $(\mathrm{r}=1 \mathrm{~cm}$ and $\mathrm{h}=5 \mathrm{~cm})$ was used. The height was reduced to $3 \mathrm{~cm}$ by additional $\mathrm{Cd}$ collars. The second step is the calibration of the sample scattering angle. This is achieved by using a polycrystalline sample. For the correct calibration, at least two Bragg-peaks are needed in each detector, but due to the small scattering angle range measurement, only one Bragg peak was possible which is enough if the wavelength is well calibrated and the distances are precisely known. The calibration of the scattering angle is needed only once since the analyzers were properly fixed against rotation around the vertical angles. To check the stability, the calibration was repeated at different energies using several standard samples: corund $\left(\mathrm{Al}_{2} \mathrm{O}_{3}\right)$, copper powder, sodiumcalcium-fluoride, etc. The standards had $21 \mathrm{~mm}$ height and $7 \mathrm{~mm}$ diameter; the powder samples were in aluminum containers. The differences from the nominal values (thus the need of calibration) are due to the small misalignment of the analyzer batches, i.e., the non perfect orientation around the vertical axis.

The calibration of the relative efficiency of the detectors is time consuming. A typical result of the vanadium measurement is shown in Fig. 10. The signal from the three PSDs of one detector unit facing one analyzer module giving the projected scattering power of the HOPG crystals is shown. Measurements with an incoherent scatterer should in principle give a locally flat signal as a function of the scattering angle. Apart from this, the scattering intensity shows a slow decrease as the scattering angle increases (see Fig. 10), which is due to the finite size of the sample. For the prototype, the horizontal size of detectors and analyzers is not matched. Therefore, intensity is measured only in the middle channels. The dip in the middle is due to the gaps between the neighboring analyzer crystals and the holes of the screws. The nominal focal point is in the middle detector (green), the higher and lower energies (lower and higher analyzer scattering angles) are shown with blue and red lines, respectively.

\section{Data treatment}

CAMEA gives a highly overlapping dataset in the 3 dimensional $q-\omega$ space due to the multiple analysed energies. The overlapping measured surfaces have different intensities and different resolutions; thus, the correct scaling of different counts is of high importance. The following method was used: The reflecting power of the analyzers together with the efficiency of the detectors is calibrated using the vanadium standard, and the elastic line of vanadium at each tube gave the pixel weight vector of the tubes. These weight vectors were multiplied by the monitor counts (projected to the sample in time) giving two dimensional weight matrices for each tube.

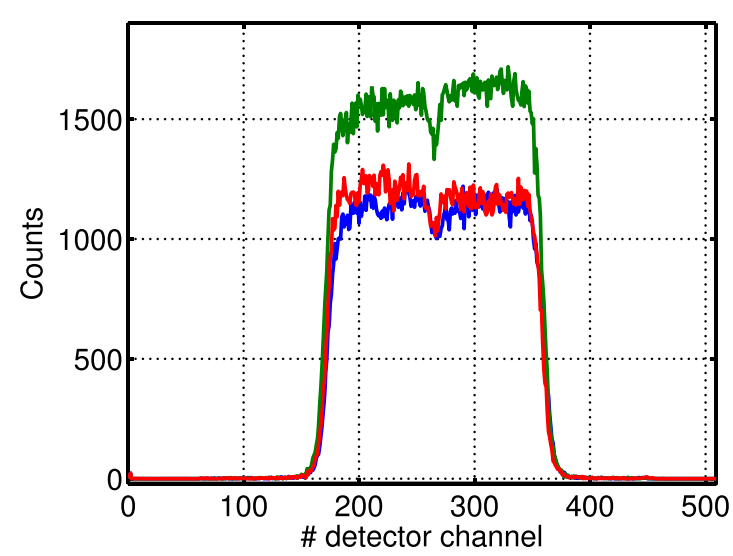

FIG. 10. Measured angular efficiency of an analyzer-detector unit: counts at different bins of the different detector tubes-blue: inner tube seeing the smaller final energy, green: middle tube, and red: outer tube seeing the larger final energy. The analyzer contained two $7.5 \mathrm{~cm}$ long crystals on one silicon wafer. In the middle, the lowered scattering efficiency of the crystals is due to the holes drilled for mounting. 
During the data reduction, counts from the detector pixels were summed in a final $q-\omega$ matrix, the same summation was applied on the weights, and finally the counts were divided by the weights. Note that this method intrinsically contains the effect of non-ideal transmission of the analyzers causing decreased intensity after the first analyzer.

\section{EXPERIMENTAL RESULTS}

\section{A. Energy resolution}

The energy resolution of the prototype was investigated by carrying out several measurements with different instrument configuration. During the measurements, the analyzer-detector geometries were fixed and the effect of analyzer mosaicity was investigated both in low and high resolution modes. The sample was vanadium with the height of $1 \mathrm{~cm}$ and $3 \mathrm{~cm}$ in high and low resolutions, respectively. The geometry and the energy resolutions can be seen in Table III.

A first measurement was performed in the low resolution mode using a solid cylindrical vanadium sample $(h=3 \mathrm{~cm}$ and $\varnothing=1 \mathrm{~cm}$ ). The geometry and resolution can be seen in the second row of Table III $\left(E_{f}=5.1 \mathrm{meV}\right)$. The whole setup was simulated using McStas. The measurement and simulation results are already published in the work of Birk et al. ${ }^{36}$ With a single overall scale factor, the simulations give an excellent description of the experimental results.

Another kind of experiment used all three analyzer modules. The instrument was operated in the high resolution mode using a second solid cylindrical vanadium sample $(h=1 \mathrm{~cm}$ and $\varnothing=1 \mathrm{~cm}$ ). The analysed energies, distances, and resolutions can be seen in Table III. The incident energy band was between $3.14 \mathrm{meV}$ and $14.86 \mathrm{meV}$. Several measurements with different analyzer mosaicities of $40^{\prime}, 60^{\prime}$, and $80^{\prime}$ were performed (see Fig. 11). Comparing the resolution as a function of mosaic spread, it is clearly visible that the mosaicity has no effect on the resolution because of the distance collimation. The experiment was repeated with an increased vanadium sample height $(h=3 \mathrm{~cm})$. In Fig. 11, the experimental data are compared to the analytical calculation of the energy resolution (solid lines in the figure), demonstrating that the resolution of the instrument can be well described. The differences between the nominal and measured energies of the elastic line are due to some alignment error of the sample height which was realized only after the data treatment.

Using HOPG as a sample, the resolution ellipsoid of the prototype was mapped in three dimensions, namely, momentum transfer in the scattering plane, $\Delta q_{x}$ and $\Delta q_{y}$ (longitudinal and transversal q-resolution), and energy transfer $\Delta E$, for

TABLE III. Energies, distances, and calculated resolutions used in the resolution measurements. In the case of resolutions, the first number shows the low resolution mode with $3 \mathrm{~cm}$ high vanadium, and the second one shows the high resolution with $1 \mathrm{~cm}$ high vanadium.

\begin{tabular}{lccccc}
\hline \hline$E_{f}(\mathrm{meV})$ & $L_{s a}(\mathrm{~m})$ & $L_{a d}(\mathrm{~m})$ & $\Delta E_{i}(\mu \mathrm{eV})$ & $\Delta E_{f}(\mu \mathrm{eV})$ & $\Delta E(\mu \mathrm{eV})$ \\
\hline 4.0 & 1.280 & 1.231 & $92 / 60$ & $76 / 38$ & $120 / 71$ \\
5.1 & 1.458 & 1.350 & $132 / 87$ & $105 / 54$ & $169 / 102$ \\
7.0 & 1.573 & 1.417 & $212 / 142$ & $170 / 89$ & $271 / 167$ \\
\hline \hline
\end{tabular}
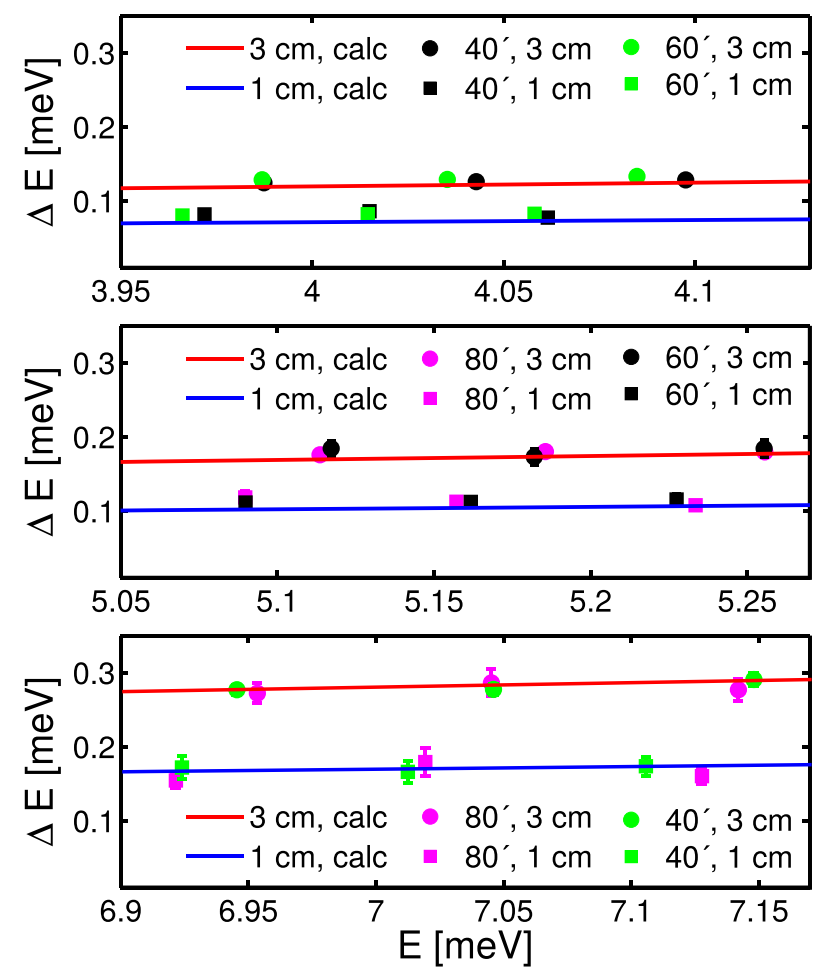

FIG. 11. Elastic energy resolution (symbols) as a function of sample height and mosaic spread of the analyzer crystals. The mosaicity has no influence on the resolution, which depends solely on the distance collimation. The results of the analytical energy resolution (solid lines) are in excellent agreement with the experimental data.

different setups with the (002) reflection. To see the whole ellipsoid of the (002) elastic peak at relatively low final energy, the analyzer energy was set to $7 \mathrm{meV}$. For each measurement, a sample rocking scan around the Bragg condition was performed resulting in three parabolic surfaces, which are close to each other in $(\mathbf{q}, \omega)$-space. This is due to the three adjacent detector tubes recording neutrons with slightly different final energies. The $q_{x}$ and $q_{y}$ directions were chosen parallel and perpendicular, respectively, to the corresponding reciprocal lattice vector. Figure 12 shows measurements done on a high quality HOPG sample (mosaicity of $0.5^{\circ}$ ) where the three dimensional data are projected to different planes, namely, $\Delta E=0, \Delta q_{x}=0$, and $\Delta q_{y}=0$. The comparison of the experimental results to the analytical calculations of the resolution ellipsoids ${ }^{28}$ shows very good agreement.

\section{B. Background}

As described in Sec. II B 3, the shielding of the prototype mainly consists of $\mathrm{Cd}$ and the main parts are walls separating the different analyzer-detector units, sidewalls (including shielding on the top and bottom of the frame) covering the three analyzer-detector units, $\mathrm{Cd}$ sheets covering the sample vacuum vessel above and below the scattering plane, and a $\mathrm{Cd}$ beam stop behind the last analyzer frame.

In order to measure the background elastic peak ratio, a standard vanadium sample with the height of $3 \mathrm{~cm}$ and diameter of $1 \mathrm{~cm}$ was used. Without any shielding, the ratio was $3 \times 10^{-2}$. Additional $\mathrm{Cd}$ walls between the different analyzer-detector units [see Fig. 13(a)] decreased the ratio 


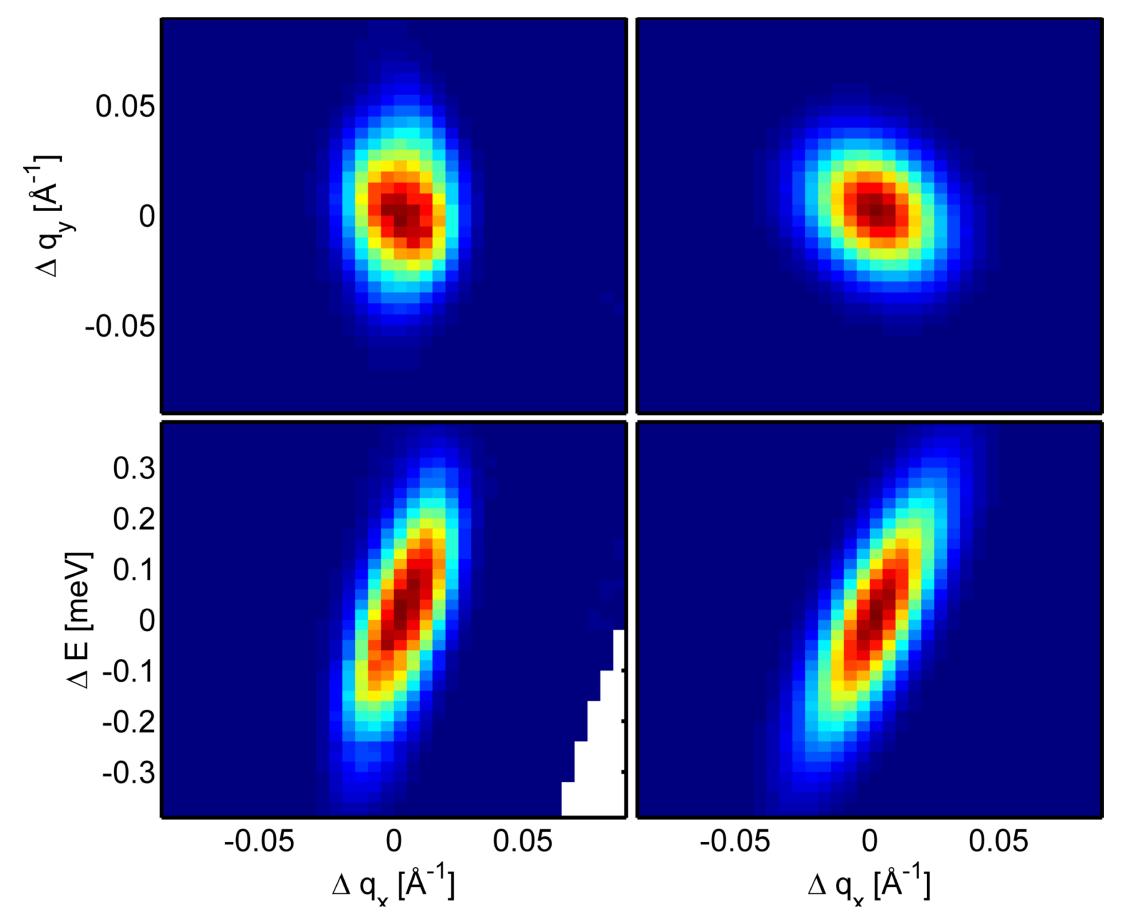

(a)

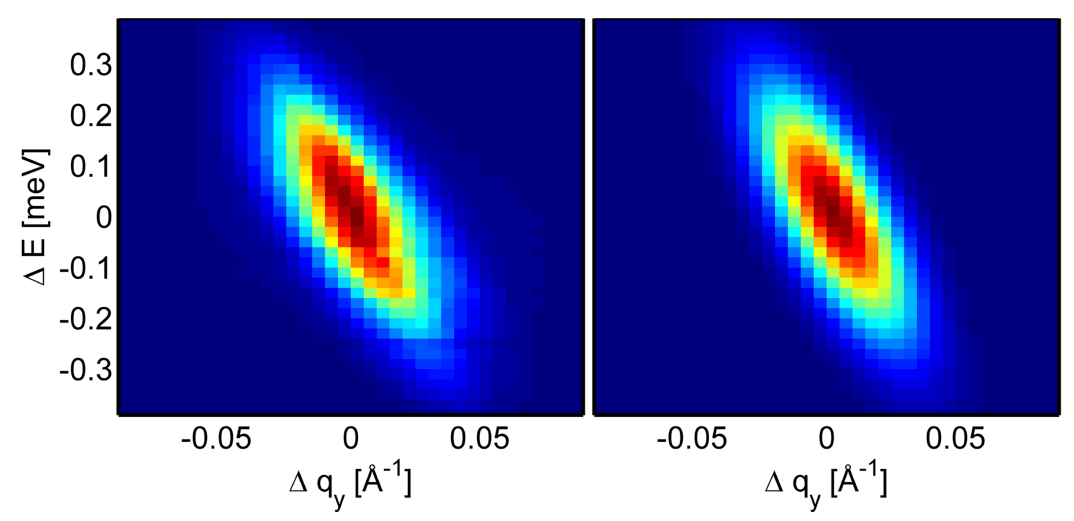

(b)
FIG. 12. Measured (left) and calculated (right) resolution ellipsoids of the prototype using a high quality HOPG sample (mosaicity of $0.5^{\circ}$ ). The sample height was $1 \mathrm{~cm}$. The final analyzer energy was set to $7 \mathrm{meV}$, and the low resolution mode was used. The experimental results are in excellent agreement with the analytical calculations. to $1 \times 10^{-2}$. Covering the sidewalls and the sample vacuum vessel above and below, the scattering plane decreased the ratio further to $4 \times 10^{-3}$. The cooling of the vanadium sample showed a strong effect: the background decreased to $3 \times 10^{-4}$ due to the decreased inelastic scattering cross section of the sample. Additional shielding in the front of the prototype

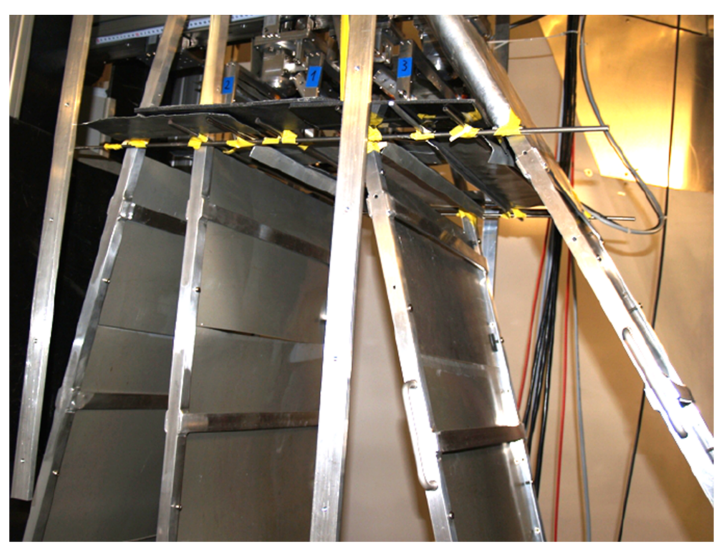

(a)

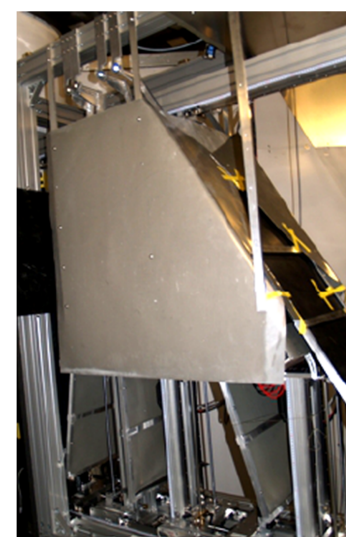

(b)
FIG. 13. Different shielding parts of the prototype. (a) $\mathrm{Cd}$ walls are inserted avoiding cross talk between different analyzer-detector channels. (b) The prototype is covered in $\mathrm{Cd}$ shielding. 


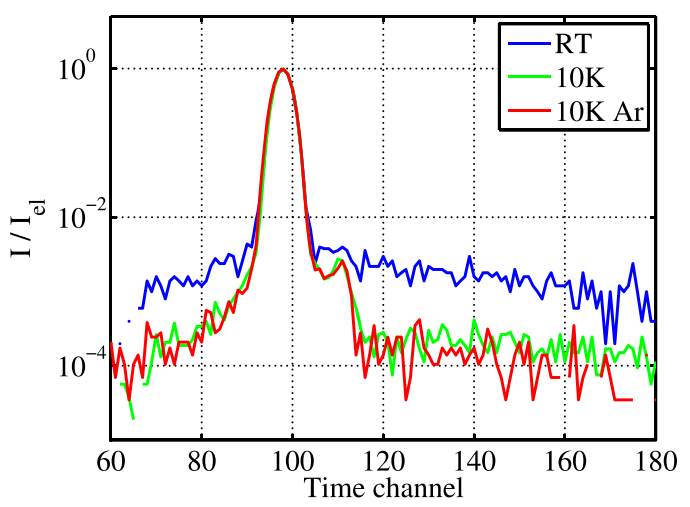

FIG. 14. Comparison of the time dependent signal of vanadium at room temperature (blue) and $10 \mathrm{~K}$ (green) and the effect of argon atmosphere in the secondary spectrometer tank. The signal is normalized to the elastic peak.

covering the unshielded steel tube of the MARS sample support and covering of the front faces of the motors and the frames of the analyzers decreased the background further below $2 \times 10^{-4}$. After the subtraction of the electronic noise, the final background was slightly above $1.2 \times 10^{-4}$. This is comparable to conventional TAS and ToF instruments. Additional measurements under argon atmosphere in the MARS secondary spectrometer tank resulted in a slight improvement of the background elastic peak ratio, which decreased below $1.2 \times 10^{-4}$. After the subtraction of the electronic background, the relative background is $5 \times 10^{-5}$. The electronic background was determined by only measuring the background between the pulses. A comparison to the intensity measured during a shutdown of the SINQ source (12.1 counts/h for one tube) was in good agreement with the intensity measured between the pulses during regular SINQ operation. As a consequence, the fast neutron background is insignificant compared to the electronic noise. The argon atmosphere increased the detected intensity by more than $20 \%$ due to the low total cross section of the argon compared to the cross section of air. Results of the background measurements for vanadium at room temperature cooled to $10 \mathrm{~K}$ and under argon atmosphere are shown in Fig. 14. This demonstrates that the intrinsically high flux at the sample (inverse ToF geometry) and the large amount of material in the beam (multiplexing) do not cause a severe background problem for the planned cold neutron spectrometers.

\section{INELASTIC MEASUREMENTS}

To demonstrate the performance of the CAMEA concept, inelastic measurements on two different samples, $\mathrm{LiHoF}_{4}$ and h- $\mathrm{YMnO}_{3}$, were carried out.

\section{A. Crystal field excitations in $\mathrm{LiHoF}_{4}$}

$\mathrm{LiHoF}_{4}$ has strong crystal field (CF) excitations at low temperatures (non-dispersive inelastic scattering). ${ }^{41}$ The sample used throughout the experiment consisted of many platelike single crystals stacked together. Measurements were done for four different temperatures in the low resolution mode at a base frequency of $14 \mathrm{~Hz}$. The measurement parameters (incident wavelength band and final energies) are the same

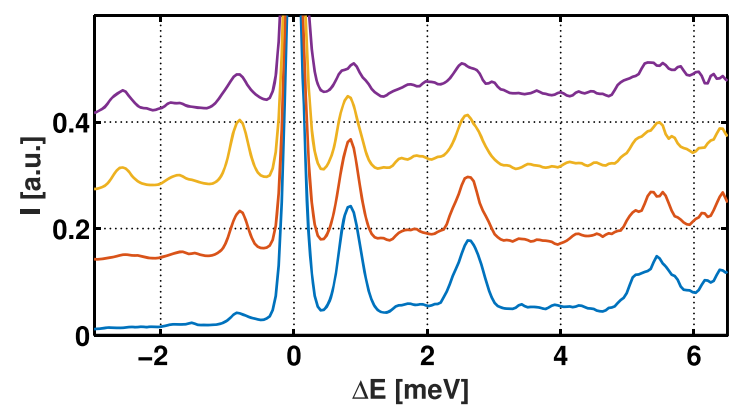

FIG. 15. Inelastic measurement on $\mathrm{LiHoF}_{4}$ using the prototype. The base lines of the four different temperature sets, $4 \mathrm{~K}$ (blue), $15 \mathrm{~K}$ (orange), $25 \mathrm{~K}$ (yellow), and $70 \mathrm{~K}$ (magenta), are shifted for the sake of visibility.

as that for the low-resolution measurements described in Sec. IV A. The sample height was $5 \mathrm{~cm}$ using a beam height of $3.2 \mathrm{~cm}$. Thus, the energy resolution was comparable. The measurement time of each temperature set was $3 \mathrm{~h}$, and the results are shown in Fig. 15. As expected, the intensity of the crystal field excitations close to $0.9 \mathrm{meV}, 2.6 \mathrm{meV}, 5.3 \mathrm{meV}$, and $6.3 \mathrm{meV}$ increases with decreasing temperature. The peak close to $-0.9 \mathrm{meV}$ increases and decreases again as the temperature decreases. This reflects the temperature dependent population of the first excited CF level. The intensity appearing between the peaks is larger than $1 \%$ of the elastic line and comes from the sample.

\section{B. Magnon dispersion in $\mathrm{h}-\mathrm{YMnO}_{3}$}

Inelastic measurements were performed of magnetic excitations in $\mathrm{h}-\mathrm{YMnO}_{3}$. The compound is spin frustrated and multiferroic. The material orders in a hexagonal structure with lattice parameters $a=b=6.18 \AA$ and $c=11.4 \AA$ below the ferroelectric transition. ${ }^{42,43}$ Below the Néel temperature $T_{N}=72 \mathrm{~K}$, $\mathrm{h}-\mathrm{YMnO}_{3}$ orders antiferromagnetically. In order to reach the Bragg peaks at the given scattering angle of the prototype $\left(2 \Theta=-60^{\circ}\right)$, higher final energies of $4.8 \mathrm{meV}, 6.1 \mathrm{meV}$, and $7.5 \mathrm{meV}$ were chosen. The single crystal sample $(m=5.2 \mathrm{~g}$, $h=8 \mathrm{~mm}$ ) was cooled to $40 \mathrm{~K}$, and the measurement was done by a sample rotation scan with a total measurement time of $20 \mathrm{~h}$. The choppers were operated in the low resolution mode, the incident energy range used was from $3.7 \mathrm{meV}$ to $20.9 \mathrm{meV}$, and the analyzers were set to the nominal analysed energies of $4.8 \mathrm{meV}, 6.1 \mathrm{meV}$, and $7.5 \mathrm{meV}$ covering a scattering angle range of $9.55^{\circ}, 8.16^{\circ}$, and $7.16^{\circ}$, respectively. The results are shown in Fig. 16. The top panel shows the measured $(\mathbf{q}, \omega)$-volume. The two bottom plots show a constant $q_{k}$-cut at $q_{k}=2.35 \AA^{-1}$ (b) and constant energy cut at $\Delta E=8 \mathrm{meV}$ (c). The excitation energies at $(\overline{2} 00)$ are in good agreement with other analytical calculations and measurements. ${ }^{43}$ Note that in these measurements only the strongest excitation is seen due to flux limitations. In the c part of the figure, it seems that the crystal is a little bit misoriented. This can be caused both by the low statistic and the non-perfect refinement (only one Bragg-peak was visible at the given range of scattering angles). The data obtained during one day on the prototype in the given $(\mathbf{q}, \omega)$-volume are equivalent to $2 \%$ of the coverage of BIFROST (ESS-CAMEA) with a 
a
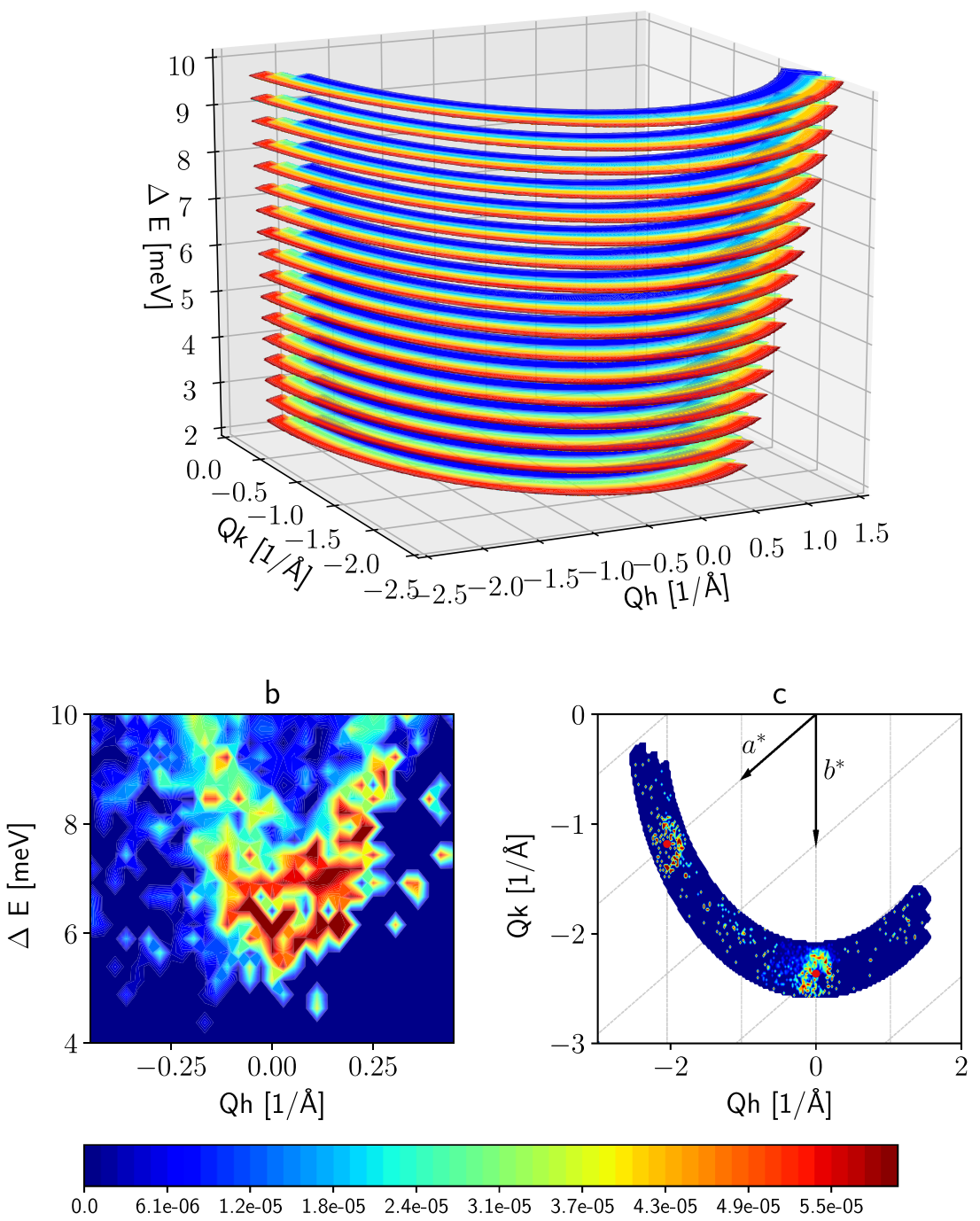

FIG. 16. Magnon dispersion of $\mathrm{h}$ $\mathrm{YMnO}_{3}$ measured with a single crystal sample at $40 \mathrm{~K}$. (a) Overview of the measured $(\mathbf{q}, \Delta E)$-volume: horizontal slices at some selected energies; the measurement gave continuous energy range. The coloring corresponds to the different detector tubes. (b) Constant $q_{k}$-cut at $q_{k}=2.35 \AA^{-1}$. The lines connecting the reciprocal lattice points (shown by red circles) are guides for eye. (c) Constant energy cut at $\Delta E=8 \mathrm{meV}$. counting time of $8 \mathrm{~s}$ applying the same resolution. Although the resolution ellipsoid is known, the low intensity did not allow investigating properly the resolution effects. This kind of investigation is reported by Toft-Petersen et al. in a similar CAMEA-like instrument (MultiFLEXX at BER II reactor, Berlin). ${ }^{26}$

\section{CONCLUSION}

A prototype of the CAMEA concept was successfully implemented and tested at SINQ, PSI, giving valuable input for the design of the planned CAMEA spectrometers at ESS and PSI. The most important results are the experimental proofs of the CAMEA concept itself and the prismatic analyzer concept. Furthermore, the analytical resolution model and the McStas simulations were validated. In both cases, the used methods were proven precise enough for further instrument optimization. The analytical model of the instrument is precise enough for the optimization of the instrument and to investigate the consequences of different geometries used. A thorough shielding resulted in a background of less than $10^{-4}$ times the elastic line of cooled vanadium. It could be shown that the optical alignment of the HOPG crystals is precise enough for orientation. This allows a much simpler aligning process of upcoming spectrometers.

\section{ACKNOWLEDGMENTS}

The authors would like to thank Finn Saxild (DTU), Ove Rasmussen (DTU), Keld Theodor (KU), Peter Keller (PSI), Christian Kägi (PSI), and Dieter Graf (PSI) for technical and engineering support and Urs Greuter (PSI) and Thomas Gahl (PSI) for detector support. This work was supported by the Danish Agency for Science, Technology and Innovation, Grant No. 11-121274, Neutron Instrumentation for the European Spallation Source design update phase, and the Swiss National Science Foundation, R’Equip Grant No. 206021-144972.

${ }^{1}$ R. Coldea, D. A. Tennant, E. M. Wheeler, E. Wawrzynska, D. Prabhakaran, M. Telling, K. Habicht, P. Smeibidl, and K. Kiefer, Science 327, 177 (2010).

${ }^{2}$ B. Lake, D. A. Tennant, J. S. Caux, T. Barthel, U. Schollwoeck, S. E. Nagler, and C. D. Frost, Phys. Rev. Lett. 111, 137205 (2013).

${ }^{3}$ H. Rønnow, R. Parthasarathy, J. Jensen, G. Aeppli, T. Rosenbaum, and D. McMorrow, Science 308, 389 (2005). 
${ }^{4}$ P. Merchant, B. Normand, K. W. Krämer, M. Boehm, D. F. McMorrow, and C. Rüegg, Nat. Phys. 10, 373 (2014).

${ }^{5}$ E. Wawrzynska, R. Coldea, E. M. Wheeler, T. Soergel, M. Jansen, R. M. Ibberson, P. G. Radaelli, and M. M. Koza, Phys. Rev. B 77, 094439 (2008).

${ }^{6}$ M. Hälg, D. Hüvonen, N. P. Butch, F. Demmel, and A. Zheludev, Phys. Rev. B 92, 104416 (2015).

${ }^{7}$ M. Bull, M. Harris, U. Steigenberger, M. Hagen, C. Petrillo, and F. Sacchetti, in 1st European Conference on Neutron Scattering (ECNS 96), Interlaken, Switzerland, October 08-11, 1996 [Phys. B 234, 1061 (1997)].

${ }^{8}$ R. Robinson, R. Pynn, and J. Eckert, Nucl. Instrum. Methods Phys. Res., Sect. A 241, 312 (1985).

${ }^{9}$ T. E. Mason, K. N. Clausen, G. Aeppli, D. F. McMorrow, and J. K. Kjems, Can. J. Phys. 73, 697 (1995).

${ }^{10}$ K. N. Clausen, D. F. McMorrow, K. Lefmann, G. Aeppli, T. E. Mason, A. Schroder, M. Issikii, M. Nohara, and H. Takagi, Phys. B 241, 50 (1997),

${ }^{11}$ K. Lefmann, D. F. McMorrow, H. M. Rønnow, K. Nielsen, K. N. Clausen, B. Lake, and G. Aeppli, Phys. B 283, 343 (2000).

${ }^{12}$ K. Lefmann, C. Niedermayer, A. B. Abrahamsen, C. R. H. Bahl, N. B. Christensen, H. S. Jacobsen, T. L. Larsen, P. Haefliger, U. Filges, and H. M. Rønnow, Phys. B 385-386, 1083 (2006).

${ }^{13}$ W. Schmidt, M. C. Rheinstaedter, S. Raymond, and M. Ohl, Phys. B 350, E849 (2004).

${ }^{14} \mathrm{~W}$. Schmidt and M. Ohl, in 8th International Conference on Neutron Scattering, Sydney, Australia, November 27-December 02, 2005 [Phys. B 385-386, 1073 (2006)].

${ }^{15}$ M. Jimenez-Ruiz, A. Hiess, R. Currat, J. Kulda, and F. J. Bermejo, Phys. B 385-386, 1086 (2006).

${ }^{16}$ O. Sobolev, R. Hoffmann, H. Gibhardt, N. Jünke, A. Knorr, V. Meyer, and G. Eckold, Nucl. Instrum. Methods Phys. Res., Sect. A 772, 63 (2015).

${ }^{17}$ F. Demmel, A. Fleischmann, and W. Glaser, Nucl. Instrum. Methods Phys Res., Sect. A 416, 115 (1998).

${ }^{18}$ F. Demmel, N. Grach, and H. M. Rønnow, Nucl. Instrum. Methods Phys. Res., Sect. A 530, 404 (2004).

${ }^{19}$ J. A. Rodriguez, D. M. Adler, P. C. Brand, C. Broholm, J. C. Cook, C. Brocker, R. Hammond, Z. Huang, P. Hundertmark, J. W. Lynn, N. C. Maliszewskyj, J. Moyer, J. Orndorff, D. Pierce, T. D. Pike, G. Scharfstein, S. A. Smee, and R. Vilaseca, Meas. Sci. Technol. 19, 034023 (2008).

${ }^{20}$ M. Kempa, B. Janousova, J. Saroun, P. Flores, M. Boehm, F. Demmel, and J. Kulda, Phys. B 385-386, 1080 (2006).

${ }^{21}$ P. G. Freeman, J. O. Birk, M. Markó, J. L. Bertelsen, N. B. Christensen, K. Lefmann, J. Jacobsen, C. Niedermayer, F. Juranyi, and H. M. Rønnow, EPJ Web Conf. 83, 03005 (2015).

${ }^{22}$ F. Groitl, D. Graf, J. O. Birk, M. Markó, M. Bartkowiak, U. Filges, C. Niedermayer, C. Rueegg, and H. M. Rønnow, Rev. Sci. Instrum. 87, 035109 (2016).

${ }^{23}$ J. Larsen, Epfl-Report-190504, 2014, http://infoscience.epfl.ch/record/ $190504 ? \ln =\mathrm{en}$.

${ }^{24}$ K. Habicht, D. L. Quintero-Castro, R. Toft-Petersen, M. Kure, L. Maede, F. Groitl, and M. D. Le, in QENS/WINS 2014-11th International Conference on Quasielastic Neutron Scattering and 6th International Workshop on Inelastic Neutron Spectrometers, EPJ Web of Conferences, edited by B. Frick, M. Koza, M. Boehm, and H. Mutka (EDP Sciences, 2015), Vol. 83.
${ }^{25}$ J. A. Lim, K. Siemensmeyer, P. Cermak, B. Lake, A. Schneidewind, and D. S. Inosov, in International Conference on Strongly Correlated Electron Systems 2014 (SCES2014), Journal of Physics Conference Series, edited by M. Zhitomirsky and P. DeReotier (IOP Publishing Ltd., 2015), Vol. 592.

${ }^{26}$ R. Toft-Petersen, F. Groitl, M. Kure, J. Lim, P. Cermak, S. Alimov, T. Wilpert, M. D. Le, D. Quintero-Castro, C. Niedermayer, A. Schneidewind, and K. Habicht, Nucl. Instrum. Methods Phys. Res., Sect. A 830, 338 (2016).

${ }^{27}$ F. Groitl, R. Toft-Petersen, D. L. Quintero-Castro, S. Meng, Z. Lu, Z. Huesges, M. D. Le, S. Alimov, T. Wilpert, K. Kiefer et al., Sci. Rep. 7, 13637 (2017).

${ }^{28}$ M. Markó, "Modular analytical calculation method for resolution function of neutron spectrometers" (unpublished).

${ }^{29}$ K. Lefmann and K. Nielsen, Neutron News 10, 20 (1999).

${ }^{30}$ L. Udby, P. K. Willendrup, E. Knudsen, C. Niedermayer, U. Filges, N. B. Christensen, E. Farhi, B. O. Wells, and K. Lefmann, in International Workshop on Neutron Optics, Grenoble, France, March 17-19, 2010 [Nucl. Instrum. Methods Phys. Res., Sect. A 634, S138 (2011)].

${ }^{31}$ P. K. Willendrup, E. B. Knudsen, E. Klinkby, T. Nielsen, E. Farhi, U. Filges, and K. Lefmann, in International Workshop on Neutron Optics and Detectors (NOP\&D), Munich, Germany, July 02-05, 2013, Journal of Physics Conference Series, edited by A. Ioffe (Forschungszentrum Julich $\mathrm{GmbH}$, Julich Ctr Neutron Sci, European Spallat Source Scandinavia, Swiss Neutron, Mirotron Ltd., S DH Heidelberg, Astrium, 2014), Vol. 528.

${ }^{32}$ P. Tregenna-Piggott, F. Juranyi, and P. Allenspach, J. Neutron Res. 16, 1 (2008).

${ }^{33}$ Principles of Optics, 7th ed., edited by M. Born and E. Wolf (Cambridge University Press, 2002).

${ }^{34}$ F. Groitl, M. Bartkowiak, R. Bergmann, J. Birk, M. Markó, A. Bollhalder, D. Graf, C. Niedermayer, C. Rüegg, and H. Rønnow, Nucl. Instrum. Methods Phys. Res., Sect. A 858, 30 (2017).

${ }^{35}$ F. Groitl, H. Kitaura, N. Nishiki, and H. M. Rønnow, "Direct bonded HOPG-Analyzer support without background source" (submitted).

${ }^{36}$ J. O. Birk, M. Markó, P. G. Freeman, J. Jacobsen, R. L. Hansen, N. B. Christensen, C. Niedermayer, M. Mansson, H. M. Rønnow, and K. Lefmann, Rev. Sci. Instrum. 85, 113908 (2014).

${ }^{37}$ M. Popovici, Acta Crystallogr., Sect. A A31, 507 (1975).

${ }^{38}$ U. Stuhr, H. Spitzer, J. Egger, A. Hofer, P. Rasmussen, D. Graf, A. Bollhalder, M. Schild, G. Bauer, and W. Wagner, Nucl. Instrum. Methods Phys. Res., Sect. A 545, 330 (2005).

${ }^{39}$ M. Markó, Building and Testing a Prototype for CAMEA, Technical Report (Paul Scherrer Institute, 2014).

${ }^{40}$ S. L. X. Peetermans, "Energy-selective neutron imaging for materials science," Ph.D. thesis, École Polytechnique Fédérale de Lausanne, 2014.

${ }^{41}$ P. Babkevich, A. Finco, M. Jeong, B. D. Piazza, I. Kovacevic, G. Klughertz, K. W. Kraemer, C. Kraemer, D. T. Adroja, E. Goremychkin, T. Unruh, T. Straessle, A. Di Lieto, J. Jensen, and H. M. Rønnow, Phys. Rev. B 92 , 144422 (2015).

${ }^{42}$ T. Katsufuji, M. Masaki, A. Machida, M. Moritomo, K. Kato, E. Nishibori, M. Takata, M. Sakata, K. Ohoyama, K. Kitazawa, and H. Takagi, Phys. Rrev. B 66, 134434 (2002).

${ }^{43}$ T. Sato, S. Lee, T. Katsufuji, M. Masaki, S. Park, J. Copley, and H. Takagi, Phys. Rev. B 68, 014432 (2003). 